\title{
A comunicação na prática médica: seu papel como componente terapêutico
}

Pedro Ribeiro da Silva*

\section{RESUMO}

A comunicação é um instrumento importante da prática médica e em especial da medicina geral e familiar, pelo seu âmbito menos específico.

A abordagem biopsicossocial para ser eficaz necessita de uma forte componente comunicacional nas diversas fases da relação médico doente, nomeadamente, na consulta, nas actividades de prevenção da doença e de educação para a saúde, na relação com os familiares do doente.

A comunicação médico-doente influencia a aderência à terapêutica pelo doente e os seus índices de satisfação com a consulta. A comunicação desempenha, também, um papel importante, conforme é adequada ou não, no desencadear ou na prevenção do stress e burnout do(a) médico(a), que são aspectos directamente relacionados com o seu bem-estar ou absentismo por doença.

A comunicação é uma componente central das estratégias de coping para as situações difíceis do quotidiano médico como as emoções associadas à doença, sofrimento e morte.

Devido ao papel fulcral que a comunicação tem na prática médica, considera-se que a formação em comunicação deve ser mais valorizada, nos diversos níveis, pré- ou pós-graduado.

Palavras-chave: Relação Médico-Doente-Família; Comunicação em Medicina Geral e Familiar; Comunicação na Consulta.

\section{INTRODUÇÃO}

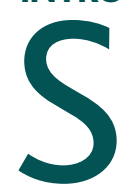

erá a comunicação uma componente importante da prática médica?

Em geral a resposta é positiva.

Mas será que o(a) médico(a) pode aperfeiçoar de forma eficaz e profunda as suas competências comunicacionais através da formação pré ou pós-graduada?

Sobre esta última questão já não existe a mesma unanimidade.

Considera-se que a facilidade em comunicar adequadamente na sociedade em geral, ou especificamente na área médica, depende de múltiplos factores como a personalidade do(a) médico(a) e a influência do seu ambiente familiar e cultural, e que as vertentes centrais que influenciam a forma de comunicar são muito difí-

*Assistente Graduado de Medicina Geral e Familiar

Divisão de Informação, Comunicação e Educação para a Saúde da Direcção-Geral da Saúde. ceis ou praticamente impossíveis de mudar.

Efectivamente, a mudança de comportamentos é, com frequência, uma tarefa difícil. Sabemos esse facto da relação com os nossos doentes quando fazemos aconselhamento sobre estilos de vida. A dificuldade em adoptar comportamentos diferentes é grande, seja deixar de fumar, fazer exercício físico ou proceder a alterações na dieta alimentar.

O mesmo acontece com as formas de comunicar. É muito difícil mudá-las.

No entanto, a prática médica tem a sua base na comunicação. A recolha da história, a transmissão de informação sobre a prescrição ou o aconselhamento sobre estilos de vida e prevenção da doença, a referenciação, o trabalho em equipa, todas estas situações que são comuns no quotidiano do(a) médico(a).

Neste artigo procede-se à análise da importância da comunicação na prática do médico de família, aprofundando-se aspectos mais complexos e menos abordados na bibliografia médica. 


\section{A CONSULTA}

Experimental studies suggest that a good history will provide $85 \%$ of the information for making a diagnosis, and examination and tests only $10 \%$ and $5 \%$ of the information; the key to a good history is good communication. ${ }^{1}$

A consulta tem uma configuração relativamente constante. Em qualquer contexto, existe a abertura da consulta, a que corresponde o primeiro contacto com o doente. ${ }^{2}$ Esta fase da consulta é muito importante porque vai influenciar todo o desenrolar da mesma. $\mathrm{O}$ seu fecho é outra fase crucial, em especial quando há continuação dos cuidados. ${ }^{2}$

A fase central da consulta permite a recolha de informação e o exame físico necessários à elaboração da história clínica, assim como na colocação de hipóteses de diagnóstico e a sua validação, que possibilitam as decisões sobre as formas de solução para os problemas do doente. ${ }^{3}$ Estes aspectos estão bem documentados na bibliografia médica que, de uma forma mais ou menos pormenorizada, explicita o papel das perguntas fechadas ou abertas, das pausas, das questões para reformulação. ${ }^{4}$ Existem vários modelos teóricos para o desenrolar da consulta, como é o caso do de Pendleton que caracteriza a existência de sete tarefas. ${ }^{5}$

Outro aspecto muito estudado, relaciona-se com a gestão do tempo. Investigações sobre o tempo de consulta têm verificado que a sua duração se situa entre dois a trinta e dois minutos, decorrendo habitualmente em cerca de oito minutos. ${ }^{6}$ Vários autores que estudaram a consulta, afirmam que interrompemos os doentes com frequência e que em média os (as) médicos (as) só esperam cerca de vinte e três segundos até à primeira interrupção, o que pode afectar a obtenção de dados importantes. $^{\text {? }}$

No entanto, vários autores têm observado que o aumento do tempo de consulta não aumenta por si só a eficácia do acto médico ou a satisfação do doente. ${ }^{8}$

Neighbour, no seu livro, descreve-nos com humor como desenvolver um estilo efectivo e intuitivo de consulta. $^{9}$

\section{A ADESÃO (COMPLIANCE) DO DOENTE ÀS PRESCRIÇÕES MÉDICAS}

Uma das áreas muito documentadas é a da aderência (compliance) dos doentes. Considera-se que não exis- te aderência quando os doentes:

- não tomam os medicamentos nas doses prescritas;

- não observam os intervalos correctos entre as doses;

- não mantêm o tratamento na duração correcta;

- excedem a dose;

- ou tomam medicamentos não prescritos.

O estudo da aderência veio sublinhar a importância da comunicação na relação médico(a)-doente e demonstrou-nos aspectos surpreendentes desta relação, nomeadamente, que os doentes com muita frequência não dizem a verdade aos seus médicos e que na sua grande maioria os médicos desconhecem a magnitude deste problema, o que origina, em média, taxas de aderência de cerca de $50 \% .{ }^{10}$ Este fenómeno tem implicações importantes sobre os resultados da prática médica, com diminuição da eficácia terapêutica, atraso no tempo de cura e aumento dos gastos em medicamentos, tratamentos e internamentos. ${ }^{11}$ Diversos estudos sobre compliance verificaram que muitos doentes, quando questionados, afirmavam ter seguido a prescrição a 100\%, mas quando foram utilizados outros métodos como a contagem de comprimidos apuraram-se taxas de adesão 2 a $59 \% .^{10}$

Quando confrontados com a não adesão, $51 \%$ dos doentes disseram que achavam não precisar da medicação, $21,7 \%$ que não a queriam tomar e $20 \%$ nem sequer a tinha comprado. ${ }^{12}$

Existem diversas razões na base da não-aderência, como a dificuldade em compreender as instruções do(a) médico(a), questões relativas à memorização (o que foi retido dessas informações) e as características psicológicas e culturais do doente.

Um dos aspectos estudados foi o tempo de espera pela consulta, tendo-se verificado que os doentes que esperaram menos de trinta minutos tiveram taxas de aderência superiores a $60 \%$, enquanto que os doentes que esperavam mais de uma hora apresentavam taxas de aderência de $30 \% .^{10}$

Outro aspecto que afecta a aderência relaciona-se com a não compreensão dos termos que o(a) médico(a) utiliza, o que acontece com maior frequência do que imaginamos. Quando, na consulta, temos dúvida se o doente compreendeu o que se disse e o questionamos sobre isso, é comum não admitirem que não compreenderam e tentarem decifrar sozinhos o que ouviram do médico a partir do seu sistema de informação, 
crenças, normas sociais e culturais. ${ }^{13}$

Os médicos, habituados a comunicar com os seus pares, usam um vocabulário específico, muito críptico, o jargão médico. ${ }^{14}$ DiMateo exemplifica com uma situação comum. Todos nós conhecemos muitas histórias como esta, a do(a) médico(a) que afirma: o Sr. tem hipertensão essencial e vou dar-lhe um medicamento para a retenção de fluidos. ${ }^{13} \mathrm{O}$ doente pensa que o comprimido é para reter os líquidos e com esse objectivo toma-o antes de deitar, para não urinar durante a noite. ${ }^{13}$

There's a gap between doctor's definition and patient' comprehension of symptoms. ${ }^{15}$

Pitts afirma:

Doctors are very poor judges of the extent of non-compliance. They are also poor at identifying which patients in particular do not comply. ${ }^{10}$

A não-aderência, é uma das áreas a que é necessário dar atenção, em especial nos doentes em que não se obtém o resultado esperado.

\section{A SATISFAÇÃO DO DOENTE}

Uma das vertentes fortemente relacionadas com a aderência é a satisfação do doente, dependendo esta de múltiplos factores, na sua maioria comunicacionais. Tate afirma ironicamente, que se fizermos as vontades ao doente ele irá de certeza ficar satisfeito. Por exemplo, aceitando fazer prescrições que os doentes nos solicitam mas que nós não consideramos adequadas. ${ }^{8}$

No entanto, efectivamente, a investigação tem reafirmado continuamente que os doentes satisfeitos melhoram mais depressa, têm maior aderência e menor consumo de consultas. Verificou-se que o tempo de consulta tem pouca influência na satisfação, mas que a comunicação, em especial o estilo de comunicação individual do(a) médico(a), tem uma grande influência.

A satisfação está fortemente correlacionada com a adequação do médico em lidar com as preocupações dos doentes, em se sentirem escutados e compreendidos, terem confiança no médico(a), bem como na expressão de sentimentos calorosos e positivos, na cortesia e na simpatia. ${ }^{8}$

A quantidade de informação influencia positivamente a satisfação, mas se o médico der pouca informação ou se esta for "excessiva», esse facto tem uma influência negativa na satisfação do doente. ${ }^{8}$

Os aspectos psicossociais e culturais são uma com- ponente importante na relação médico(a)-doente com grande influência na satisfação.

\section{VERTENTE PSICOSSOCIAL E CULTURAL}

O treino médico privilegia a objectividade, a doença física, dando menos importância ao contexto psicossocial, considerado mais subjectivo.

Muitos dos doentes têm idades, estudos e vivências muito diferentes da do médico(a), o que é um factor de dificuldade de comunicação.

As consultas feitas a doentes com idade, estudos e culturalmente mais próximos do médico(a) são em regra mais longas, fenómeno que a Psicologia Social define como homofilia, em que considera ser a comunicação mais efectiva quando os interlocutores partilham o mesmo sistema de significados, representações sociais, crenças, valores, estereótipos e preconceitos.

Para Vaysse, esta situação agravou-se a partir dos anos 80 do século XX:

- The value systems for patients and doctors seem more divergent than before. ${ }^{15}$

Esta diferença de conhecimentos, vivências, valores e crenças, torna-se um obstáculo à compreensão mútua e às possibilidades de empatia.

Empatia consiste na capacidade de uma pessoa aceder ao que outra sente e pensa no momento em que estão em presença uma da outra, interpretando e sentindo esses pensamentos como se fossem seus, sem perder a sua identidade. ${ }^{16}$ Compreender e sentir a experiência do doente, as suas necessidades, ansiedades, alegrias, desgostos e mágoas como se fossem suas.

A empatia é um instrumento fundamental para a comunicação entre o profissional de saúde e o doente e deve ser treinada e aperfeiçoada, porque se é complexa com uma pessoa semelhante, torna-se extremamente difícil com uma pessoa muito diferente em idade ou estatuto sócio-cultural.

Este facto torna-se patente quando o(a) médico(a) fica doente. Caprara relata-nos o caso de um médico endocrinologista com esclerose lateral amiotrófica que consultou um especialista e que posteriormente descreveu esse encontro num livro que publicou:

Fiquei desiludido com a maneira impessoal de se comunicar com os pacientes. Não demonstrou em momento nenhum interesse por mim como pessoa que estava sofrendo. ${ }^{17}$ Alguns meses depois desse contacto decep- 
cionante, o mesmo médico, lendo um artigo escrito pelo colega especialista que tinha consultado, ficou surpreendido diante da importância que o mesmo atribuía ao papel do suporte moral e psicológico no tratamento de pacientes com esclerose lateral amiotrófica. ${ }^{17}$

\section{A ABORDAGEM BIOPSICOSSOCIAL}

A menor valorização da componente psicossocial não afecta apenas a relação médico(a)-doente mas também a leitura clínica das vertentes psicossociais e emocionais da doença e a dificuldade de lidar com as situações que não são claras do ponto de vista físico e biológico. ${ }^{18}$ Nestas situações, atribui-se ao doente o rótulo de psicossomático, o que pode contribuir para diminuir a eficácia do acto médico e criar dificuldades na relação com o doente.

Os padrões teóricos da biomedicina valorizam os factores etiológicos externos (v.g. químicos, ambientais e comportamentais) considerados numa perspectiva de causa-efeito linear, basicamente biológica.

Às componentes psicossociais é atribuída uma importância muito diminuta. A psicossomática tem sido a excepção, mas tem tido dificuldade em impor-se e restringe-se à componente psicológica, não colocando em evidência as vertentes socioculturais na etiologia da doença.

As amigdalites, diarreias ou dores abdominais de repetição de uma criança, podem estar relacionadas com um problema escolar ou familiar. Prescrever medicamentos resolve apenas temporariamente as consequências, sem actuar sobre as causas que são inicialmente psicossociais.

O equilíbrio biológico depende da harmonia ao nível psicológico e esta depende dos contextos relacionais e culturais.

O sistema imunológico está directamente implicado e tem relações estreitas com os outros sistemas, nomeadamente o neuro-endócrino. Por sua vez, os estados psicológicos e emocionais estão intimamente relacionados com estes sistemas e são interdependentes dos factores sociais e culturais.

Uma comunicação adequada com o doente permite perceber, com alguma facilidade, estas interdependências e tornar mais eficaz a nossa prática, mudando da perspectiva biomédica para a abordagem psicossocial.

Podemos considerar, que para o médico de família praticar uma abordagem psicossocial, que é o centro teórico da sua actuação, é importante ter os conhecimentos e desenvolver as capacidades que se relacionam com esse tipo de abordagem. Mas se não comunicar adequadamente com o doente terá dificuldade em fazer a avaliação e o diagnóstico das componentes psicossociais da doença, resumindo a sua actuação à componente biológica da patologia.

Com frequência, não damos tempo ao doente para exprimir sentimentos, angústias, dificuldades familiares ou socioculturais. Somos sintoma-centrados, focalizados na doença física.

Queremos saber o máximo sobre o doente mas pouco sobre a pessoa. ${ }^{8}$

\section{RELAÇÃO}

A relação entre médico e doente é complexa e por vezes difícil, podendo ser até conflituosa.

Parte da dificuldade da relação advém de serem com frequência pessoas tão diferentes ao nível da idade, estatuto sociocultural, vivências. Têm visões da realidade diferentes e com frequência os objectivos que desejam alcançar através da consulta são diferentes ou até opostos. Ao conjunto de expectativas, interesses e objectivos, tem-se atribuído a denominação de agenda. Médicos e doentes têm na maior parte das vezes agendas diferentes. Este facto não seria problemático se o doente explicitasse a sua agenda ao médico e se tivesse um conhecimento da consulta e do seu desenrolar o mais próximo possível daquela que tem o médico. Na realidade existe uma grande assimetria na relação médico-doente, pois o médico tem os conhecimentos e domina o ritual da consulta. As agendas ou parte das agendas são escondidas do médico, o que dificulta a relação e cria possibilidades de conflito de interesses e de relação. ${ }^{19}$

Tate considera que só quatro de 35 doentes mencionam toda a sua agenda durante a consulta. ${ }^{8}$

Nestes casos, a continuação de cuidados inerente à medicina familiar pode ser paradoxal. Se, nuns casos, a continuação permite ir aumentando a confiança que o doente tem no médico e dessa forma ir conhecendo a sua agenda escondida, o que contribui para melhorar a relação e tornar mais eficaz a prática médica, noutros casos a continuação dos cuidados pode agravar uma situação que se torne conflituosa. Por diversas ra- 
zões, o conflito com o doente é um aspecto importante da prática médica, das quais salientamos o stress e o burnout associados à prática da medicina familiar e o aumento da frequência de participações e processos dirigidos a médicos pelos doentes.

Em países como os E.U.A., onde tem aumentado o número de médicos processados por doentes, quando se estudam as razões para este facto, nem sempre se encontra como causa um erro médico de diagnóstico ou de terapêutica, mas questões ligadas com a relação médico-doente.

Diversos estudos mostram percentagens de $20 \%$ de doentes adultos que referem ter dificuldades de comunicação com os seus médicos. Este facto representa um enorme manancial de probabilidade de participação ou processo contra os médicos. ${ }^{20}$

Um dos aspectos a trabalhar na relação com o doente relaciona-se com o estabelecimento claro dos limites da relação entre médico e doente.

Tanto quanto possível, logo no início da relação deve ser negociado o conjunto de regras que a vão nortear. Se esses limites não forem negociados e estabelecidos claramente, o doente pode invadi-los ao nível do espaço e do tempo, muitas vezes através de amigos e da família ou da oferta de prendas. ${ }^{21}$

Os limites também podem ser ultrapassados pelo médico quando permite que os seus sentimentos pessoais invadam a prática ou quando comenta com terceiros, mesmo que familiares e sem autorização ou conhecimento do doente, aspectos que são sigilosos. ${ }^{22}$

\section{STRESS E BURNOUT MÉDICO}

O exercício profissional da medicina implica uma forte componente de stress emocional, que é constante. Diariamente se contacta com a doença, o sofrimento e a morte e se tem que dar más notícias a doentes e familiares.

Este acumular de stress profissional pode provocar o esgotamento e burnout do médico. O stress persistente e repetitivo, que se instala rotineiramente, pode ter um efeito devastador sobre o médico, quando após meses ou anos de instalação silenciosa se organiza como sintoma físico que, entre muitas outras possibilidades, pode ser gastrite, úlcera, insónia, cefaleias de repetição, taquicardia, hipertensão arterial, distúrbios gastro-intestinais, problemas dermatológicos, que po- dem desencadear uma situação de burnout psicológico ou de doença física grave..$^{23,24}$

Uma das principais causas de stress e de burnout médico não é o excesso de trabalho, mas fundamentalmente as situações em que o médico sente que não está a ser eficaz e que são com frequência situações relacionadas com aspectos de comunicação interpessoal, como a não aderência dos doentes às prescrições, relações difíceis ou mesmo conflitos com os doentes ou colegas de trabalho.

As dificuldades de lidar com as emoções relacionadas com a doença e a morte, podem enquadrar-se no âmbito da comunicação intrapessoal e podem ser diminuídas ou resolvidas através do treino das estratégias de coping individual com estas situações. Estas estratégias de coping são, na sua maioria, estratégias de comunicação intrapessoal, que é o primeiro patamar da comunicação (como Pedro Frade bem explicita ${ }^{25}$ ), e que relaciona o indivíduo com as suas expectativas relativamente à realidade e com a gestão interna das emoções. As estratégias de coping são também importantes para gerir o stress causado pela rotina do trabalho médico.

Quando a prática médica corre bem, o que comporta um conjunto de emoções positivas protectoras do estado anímico e psicológico, mesmo trabalhando em excesso existem menos possibilidades de haver stress patológico, que advêm, na sua maioria, das situações que não se conseguem controlar, em especial doentes particularmente difíceis, frequentadores assíduos com problemas insolúveis, conflitos com doentes ou com colegas. As mudanças forçadas, frequentes ou mal integradas, nas formas e condições de trabalho, são também um forte factor de stress profissional.

Quando o médico não consegue gerir o stress, que se acumula em excesso e começa a provocar esgotamento, cria-se um círculo vicioso que por vezes é difícil de quebrar. O médico em stress e esgotado fica com menos interesse pela consulta e pelos doentes, fica também com menos capacidade de lidar com problemas e irrita-se mais facilmente. Resumindo, piora a sua capacidade de comunicação, o que pode aumentar o stress e o burnout.

Investigações demonstram que os médicos em stress e com pouca satisfação no trabalho, prescrevem mais medicamentos, nomeadamente tranquilizantes. ${ }^{8}$ 
Os grupos Balint, ou outro tipo semelhante de grupos de médicos que se encontrem periodicamente para reflectirem nestes aspectos da comunicação na consulta, podem ser óptimos instrumentos para os médicos aperfeiçoarem as suas capacidades de comunicação com os doentes e de melhorarem as suas estratégias de coping com o stress emocional e profissional da prática médica. ${ }^{26-28}$

\section{PREVENÇÃO DA DOENÇA, EDUCAÇÃO E PROMOÇÃO DA SAÚDE}

A prevenção da doença e a educação para a saúde são duas áreas importantes da medicina familiar, em especial devido ao aumento de pessoas com doença crónica. No entanto, as taxas de sucesso poderiam ser mais elevadas se as metodologias e formas de comunicação utilizadas fosem mais adequadas. ${ }^{29}$

Todos conhecemos a dificuldade de grande parte dos doentes em adoptarem estilos de vida protectores da saúde, como a actividade física, a diminuição ou paragem do consumo de tabaco, dormir o suficiente, evitar comer determinados alimentos, prevenir os acidentes, aprender a gerir o stress, promover o envelhecimento saudável. ${ }^{30}$

As iniciativas de prevenção da doença e educação para a saúde são na sua essência actividades sustentadas na comunicação com o doente.

A adequação dos processos comunicacionais aos diferentes doentes e aos seus diversos contextos socioculturais, é um dos principais instrumentos para aumentar a eficácia das intervenções de prevenção da doença e educação para a saúde.

Os comportamentos das pessoas têm origem em vivências e situações contextuais de grande complexidade, onde se incluem as componentes culturais, sociológicas e psicossociais, que são muito diferentes de indivíduo para indivíduo e com frequência muito diferentes das do médico.

Os doentes justificam as suas opções comportamentais com informações e crenças que, não sendo científicas na perspectiva da biomedicina, se tornam por vezes muito difíceis de debater.

Para se ser eficaz ao nível dos comportamentos e estilos de vida dos utentes, é necessário conhecer as suas características individuais de modo a encontrar-se uma estratégia adequada e eficaz para cada pessoa.
Nesta vertente da prática da medicina familiar, prevenção da doença e educação para a saúde, é importante aprofundar os conhecimentos e as capacidades comunicacionais nas vertentes acima inventariadas: culturais, sociológicas e psicossociais.

\section{O CORE DA COMUNICAÇÃO MÉDICO-DOENTE}

Todas as situações abordadas anteriormente integram a relação médico-doente e têm como traço comum uma componente comunicacional importante. Foram analisadas separadamente porque cada uma apresenta especificidades muito próprias, que devem ser reflectidas quando se faz formação sobre a comunicação e a relação médico-doente.

No entanto, sempre que um médico e um doente ou familiar estão em presença, independentemente do estatuto que cada um tem no momento, estamos perante uma situação comum de comunicação com tudo o que lhe é inerente.

O primeiro contacto é fulcral para o estabelecimento da relação.

O que mais influencia esse primeiro contacto é a comunicação não verbal, nas suas diversas linguagens: postura corporal, gestos, mímica facial, sorriso, contacto ocular, voz nas suas diversas dimensões, vestuário, estatura, cabelo, adereços (óculos, outros adereços), higiene, perfume. Estão identificadas mais de 80 linguagens não-verbais.

Normalmente privilegiamos a linguagem verbal, mas esta serve essencialmente para transmitir conteúdos e informação.

É a linguagem não-verbal que transmite sentimentos e emoções e estabelece a relação.

Num primeiro contacto com uma pessoa que não se conhece, a nossa resposta depende muito mais da análise que fazemos do seu não-verbal do que do efeito que possa ter o que nos disse verbalmente.

Porque é mais difícil mentir ou ocultar estados de espírito através do não-verbal.

Neste sentido, devemos ser cuidadosos com a primeira impressão, que se torna fulcral na relação futura, pois é árduo conseguir mudar uma primeira impressão, em especial se for negativa.

Como podemos então melhorar a nossa comunicação não-verbal e torná-la mais eficaz?

Efectivamente, esse é um dos problemas que só a 
formação adequada pode resolver.

Consideremos apenas duas das linguagens não-verbais a que se pode atribuir um papel central na relação interpessoal: a voz e a mímica facial.

A própria pessoa tem muito pouco ou quase nenhum controlo sobre a sua voz e a sua mímica facial. Quando gravamos a voz, ou nas fotos e filmes, normalmente existe uma certa estranheza com o que ouvimos ou observamos. ${ }^{31}$

Daí, ser tão difícil, por exemplo, a uma pessoa que tem uma voz ou um fácies agressivos, mudar.

A formação através de filmagens, permite observar e posteriormente trabalhar estas características de comunicação e adequá-las às diversas situações do quotidiano.

Mas, se não se fizer formação nesta área com professores experientes, é muito possível manter-se durante toda a vida um estilo de comunicação inadequado, sem disso se ter consciência e portanto sem se perceber porque existe maior dificuldade de comunicação e é gerado um maior número de conflitos com as outras pessoas.

Outro aspecto do core comunicacional relaciona-se com a expressão das emoções. Existem emoções que são protectoras da saúde e emoções que lhe são nocivas. ${ }^{32}$ Quando comunicamos, estamos a transmitir emoções. No caso do médico, este aspecto é fundamental, pois transmitir as emoções positivas para o doente é a principal vertente do efeito placebo que o médico pode constituir para o doente - efeito psicológico provocado pela forma de comunicar. ${ }^{33}$

Mas, para que isso aconteça, é necessário que o médico perceba e conheça as emoções que está a transmitir, o que não é fácil, necessitando de treino e aprendizagem. Na sua essência, consiste em aprofundar a comunicação intrapessoal, aprender a conhecer o seu self e respectivas manifestações.

Outra vertente da comunicação interpessoal a valorizar é a de que duas pessoas quando estão em presença sincronizam. É essa, aliás, a essência da comunicação. Sincronizam gestos, intensidade de voz, ritmo de fala, posição do corpo, expressão facial. ${ }^{34}$

Esta sincronização é automática, não reflectida por nenhum dos interlocutores, sendo mais fácil com pessoas semelhantes e mais difícil com pessoas muito diferentes. No entanto, estando completamente desvalo- rizado na formação médica, é um dos aspectos a observar, reflectir e aprofundar, na formação dos médicos em comunicação.

Fizemos uma análise muito sumária de vários aspectos que nos parecem importantes na relação médico(a)-doente. Ficaram por analisar outras componentes desta relação, como a utilização do espaço, disciplina denominada por Proxémia, o modelo das crenças de saúde, os problemas relacionados com a transculturalidade, a referenciação e os aspectos éticos.

Como conclusão, consideramos a comunicação como um instrumento central para uma prática médica eficaz.

A necessidade de formação em comunicação adequada à prática profissional dos médicos é inquestionável.

Porque não existe um modelo único de comunicação que sirva para todos os médicos.

Pour poursuivre une discussion constructive sur les relations médecin-patient, nous proposons de discuter d'un répertoire de relations plutôt que d'insister sur un seul type de relations dans toutes les situations cliniques. La variation des relations entre médecin et patient est à prendre en compte dans diverses circonstances. ${ }^{35}$

\section{ENDEREÇO PARA CORRESPONDÊNCIA:}

Pedro Ribeiro da Silva

E-mail: pedros@dgs.pt

\section{REFERÊNCIAS BIBLIOGRÁFICAS}

1. McManus IC. Psychology in medicine. Oxford: Butterworth-Heinemann; 1992.

2. Hargie O, editor. A handbook of communication skills. London: Routledge; 1986.

3. Tanner BA, editor. Language and communication in general practice. London: Hodder and Stonghton; 1976.

4. Dickson DA, Hargie OW, Morrow NC. Communication skills training for health professionals. London: Chapman \& Hall; 1989.

5. Pendleton D, Schofield T, Tate P, Havelock P.A consulta: uma abordagem à aprendizagem e ensino. Porto: Departamento de Clínica Geral - FMUP; 1993.

6. Tuckett D, Bolton M, Olson C, Williams A. Meetings between experts. New York: Tavistock Publications; 1985.

7. Travaline JM, Ruchinskas R, D'Alonzo GE Jr. Patient-physician communication: why and how. Jthe Am Osteopath Assoc 2005 Jan; 105 (1): 13-8.

8. Tate P. Guia prático da comunicação médico-doente. Lisboa: Climepsi; 2004.

9. Neighbour R. The inner consultation. Lancaster: MTP Press; 1987.

10. Pitts M. The psychology of health. London: Routledge; 1991. 
11. Task Force for Compliance. Noncompliance with medications: an economic tragedy with important implications for health care reform. Baltimore, Md: Task Force for Compliance; 1994.

12. Lowes R. Patient-centered care for better patient adherence. fam Pract Manag 1998 Mar; 5 (3): 46-7, 51-4, 57.

13. DiMatteo MR, DiNicola DD. Achieving patient compliance. New York: Pergamon Press; 1982.

14. Baylon C, Mignot X. La communication. Paris: Nathan Université; 1991.

15. Vaysse J. Culture and communication in medicine. Symposium Family and Culture in General Practice - The European Approach; 1994 May 25-28; Lisboa, Portugal. Paris; 1994.

16. Blondis MN, Jackson BE. Nonverbal communication with patients. New York: John Wiley \& Sons; 1982.

17. Caprara A, Lins A, Franco S. A relação paciente-médico: para uma humanização da prática médica. Cad Saúde Pública 1999 Jul-Set; 15 (3): 647-54.

18. Weinman J. An outline of psychology as applied to medicine. 2nd ed. Bristol:Wright; 1987.

19. Faulkner A. Effective interaction with patients. London: Churchill Livingstone; 1992.

20. Audil NA, Shaw JD. Policy white paper - Malpractice impacts: communication between patient and physician. New York 2004 Oct; 1-3.

21. Douglas J. How to treat your doctor. London: Bloomsbury Publishing; 1992.

22. Salinsky J, Sackin P. Médicos com emoções: identificar e evitar comportamentos defensivos na consulta. Lisboa: Fundação Grunenthal; 2004.
23. Gatchel RJ, Baum A, Krantz DS. An introduction to health psychology. 2nd ed. New York: McGraw-Hill; 1989.

24. Leal MF. Stress e burnout. Porto: Laboratórios Bial; 1998.

25. Carrilho MM, Sàágua J. Dicionário do pensamento contemporâneo. Lisboa: Edições D. Quixote; 1991.

26. Balint M. O médico, o seu doente e a doença. Lisboa: Climepsi Editores; 1998.

27. Balint E, Norell JS, editors. Six minutes for the patient: interactions in general practice consultation. London: Tavistock/ Routledge; 1973.

28. Brandão J. Relação médico-doente: sua complexidade e papel dos grupos Balint. Rev Port Clín Geral 2007 Nov-Dez; 23 (6): 733-44.

29. Silva PR. A educação para a saúde e o marketing social. In: Dias MR, Durá E, editores. Territórios da psicologia oncológica. Lisboa: Climepsi 2002. p. 189-211.

30. Vickers KS, Kircher KJ, Smith MD, Petersen LR, Rasmussen NH. Health behaviour counselling in primary care: provider-reported rate and confidence. Fam Med 2007 Nov-Dec; 39 (10): 730-5.

31. Oliver RW. Psychology and health care. London: Baillière Tindall; 1993.

32. Takemura YC, Atsumi R, Tsuda T. Which medical interview behaviours are associated with patient satisfaction? Fam Med 2008 Apr; 40 (4): 253-8.

33. Loxterkamp D. A friend in need. BMJ 2008 Jul 5; 337 (7660): 30-1.

34. Niven N. Health psychology: an introduction for nurses and other health care professionals. London: Churchill Livingstone; 1989.

35. Lussier MT, Richard C. En l'absence de panacée universelle: répertoire des relations médecin-patient. Can Fam Physician 2008 Aug; 54 (8):1096-9.

\footnotetext{
ABSTRACT

Communication is a very important tool on medical practice, especially in Family Medicine and Primary Care because of its broad scope.

To be effective, the biopsychosocial approach needs to rely on a very strong communicational component during the different stages of the doctor-patient relationship, particularly the medical appointment, disease prevention activities, health education and when contacting with the patient's relatives.

The doctor-patient communication can exert an influence on the compliance of the patient and his satisfaction with the medical appointment. Communication also has an effect on the physician; when it is adequately conveyed it can prevent stress or burnout problems, improving his well-being as well as reducing absenteeism.

Communication plays a key role in the strategies of coping with hard situations in the daily medical practice, such as facing the emotions brought about by disease, death and pain.

Because of the great significance of communication in medical practice it should play a bigger role in the different levels of medical education, both during graduation and post-graduation.
}

Keywords: Physician-Patient-Family Relation; Communication in Family Medicine; Communication in the Medical Appointment. 